\title{
Goi3-dependent inhibition of JNK activity on intracellular membranes
}

\author{
Guillaume Bastin, Jin Ye Yang and Scott P. Heximer* \\ Department of Physiology, Heart and Stroke, Richard Lewar Centre of Excellence in Cardiovascular Research, University of \\ Toronto, Toronto, ON, Canada
}

Heterotrimeric G-protein signaling has been shown to modulate a wide variety of intracellular signaling pathways, including the mitogen-activated protein kinase (MAPK) family. The activity of one MAPK family class, c-Jun N-terminal kinases (JNKs), has been traditionally linked to the activation of G-protein coupled receptors (GPCRs) at the plasma membrane. Using a unique set of G-protein signaling tools developed in our laboratory, we show that subcellular domain-specific JNK activity is inhibited

OPEN ACCESS

Edited by:

Richard Neubig,

Michigan State University, USA

Reviewed by:

David L. Roman,

The University of lowa, USA Joe B. Blumer, Medical University of South Carolina,

USA

*Correspondence:

Scott P. Heximer,

Department of Physiology, Heart and Stroke, Richard Lewar Centre of Excellence in Cardiovascular Research, University of Toronto, 1 King's College Circle, Toronto,

ON M5S 1A8, Canada

scott.heximer@utoronto.ca

Specialty section:

This article was submitted to

Computational Physiology and Medicine, a section of the journal Frontiers in Bioengineering and Biotechnology

Received: 03 June 2015

Accepted: 13 August 2015 Published: 01 September 2015

Citation:

Bastin G, Yang JY and Heximer SP (2015) Gai3-dependent inhibition of JNK activity on

intracellular membranes.

Front. Bioeng. Biotechnol. 3:128. doi: 10.3389/fbioe.2015.00128 by the activation of $\mathrm{G} \alpha \mathrm{i}$, the Goi isoform found predominantly within intracellular membranes, such as the endoplasmic reticulum (ER)-Golgi interface, and their associated vesicle pools. Regulators of intracellular Goi3, including activator of G-protein signaling 3 (AGS3) and the regulator of G-protein signaling protein 4 (RGS4), have a marked impact on the regulation of JNK activity. Together, these data support the existence of unique intracellular signaling complexes that control JNK activity deep within the cell. This work highlights some of the cellular pathways that are regulated by these intracellular complexes and identifies potential strategies for their regulation in mammalian cells.

Keywords: Galphai3, JNK mitogen-activated protein kinases, AGS3, RGS proteins, intracellular membranes

\section{Introduction}

Heterotrimeric G-proteins function as molecular switches to regulate intracellular signaling pathways. The timing and duration of these signals are dependent on the lifetime of the activated (GTP-bound) G $\alpha$ subunit. The conventional model for receptor-mediated G-protein activation is described herewith. In the basal state, a quiescent (GDP-bound) G $\alpha$ subunit is complexed with a G $\beta \gamma$ heterodimer and coupled to the intracellular surface of a GPCR. Receptor activation by an extracellular stimulus results in the exchange of GTP for GDP on the G $\alpha$ subunit and the

Abbreviations: AGS, activator of G-protein signaling; AP-1, activator protein 1; ATF2, activating transcription factor 2; BAD, $\mathrm{Bcl} 2$-associated death promoter; $\mathrm{Bcl} 2$, B-cell lymphoma 2; BIM, Bcl2 interacting mediator of cell death; CFP, cyan fluorescent protein; DHHC, Asp-His-His-Cys containing motif palmitoylating enzyme; DHHC, palmitoyl-CoA transferase active site motif; EBSS, Earle's balanced sodium solution; ER, endoplasmic reticulum; ERGIC, ER-Golgi intermediate compartment; FOXO4, forkhead box protein O4; GAIP, G alpha interacting protein; GAP, GTPase-activating protein; GDI, guanosine nucleotide dissociation inhibitor; GDP, guanosine-diphosphate; GEF, guanine nucleotide dissociation inhibitor; GFP, green fluorescent protein; GPCR, G-protein coupled receptor; G-protein, guanine nucleotide binding protein; GTP, guanosine5,-triphosphate; HEK, human embryonic kidney; JNK, c-Jun N-terminal kinase; MAPK, mitogen-activated protein kinase; MEKK, mitogen-activated protein/ERK kinase kinases; MKK, mitogen-activated protein kinase kinase; MKK4/7, mitogenactivated protein kinase kinase 4/7; PI3K, phophoinositide 3-kinase; PM, plasma membrane; RFP, red fluorescent protein; RGS, regulator of G-protein signaling; TGN, trans-Golgi network; WT, wild type; YFP, yellow fluorescent protein. 
dissociation of GTP-bound G $\alpha$ from the G $\beta \gamma$. This condition marks the activated ("ON") state of receptor signaling, during which time the $G \alpha$ and $G \beta \gamma$ subunits are free to engage downstream effector molecules, such as adenylyl cyclases, phospholipases, and ion channels. Effector signaling is terminated following $\mathrm{G} \alpha$-catalyzed hydrolysis of GTP and reformation of the quiescent ("OFF") state of the GPCR complex. Based on their membranespanning nature and their ability to transmit a wide array of extracellular signals to appropriate intracellular effector pathways, GPCRs represent one of the most important classes of therapeutic targets for drug discovery (Hopkins and Groom, 2002).

A less-well characterized pathway for the activation of G-protein $\alpha$ subunits involves regulatory proteins that reside on intracellular membranes. Some $\mathrm{G} \alpha$ subunits, such as $\mathrm{G} \alpha \mathrm{i} 3$, for example, are localized primarily to intracellular membrane pools like the trans-Golgi network (TGN) (De Vries et al., 1998), LC3-positive autophagosomes (Garcia-Marcos et al., 2011), and the ERGIC, a trafficking compartment between the ER and the Cis-Golgi (Lo et al., 2015). A number of intracellular proteins can regulate the activity of Goi3 at sites distal to the plasma membrane receptor complexes discussed above. G-protein dissociation inhibitors (GDIs) maintain the inactive signaling conformation of the Gai/o subunits by stabilizing the GDPbound state (Siderovski and Willard, 2005). One of the best known GDIs is AGS3, a protein found primarily on intracellular membranes at the Golgi, aggresomes, and LC3-positive autophagosomes (Vural et al., 2010; Garcia-Marcos et al., 2011; Oner et al., 2013). AGS3 can also prime Goi/o subunits for activation by non-receptor guanine nucleotide exchange factors (GEFs), such as GIV (Lo et al., 2015). Importantly, GIV was

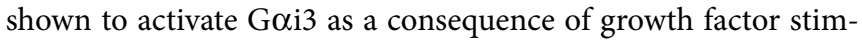
ulation, supporting the premise that G $\alpha \mathrm{i} 3$ can be activated in a receptor-independent manner. Notably, GIV was found to be localized to the same compartments as Goi3 including the plasma membrane, ERGIC, TGN, and AGS3-labeled autophagosomes (Garcia-Marcos et al., 2011; Lo et al., 2015). Another family of $\mathrm{G} \alpha$ subunit regulators, the RGS protein superfamily, functions as GTPase-activating proteins (GAPs) to inhibit Gprotein signaling (Berman et al., 1996). There are more than 35 proteins in the human genome. All RGS proteins are typified by their $\sim 120$ aa GAP domain that is capable of increasing the rate of GTP hydrolysis by up to 2000 -fold. They can be further classified into subgroups based on the organization of multiple modular protein-protein interaction domains that direct their localization to specific signaling complexes within the cell (Hollinger and Hepler, 2002). Like Goi3, AGS3, and GIV, some RGS proteins are concentrated within intracellular membrane domains. Specifically, RGS19 is localized mainly to the TGN and plasma membrane where it can regulate Goi3mediated control of protein sorting and autophagic flux. We have previously shown that RGS4, a member of the regulators of Gprotein signaling (RGS) protein superfamily, can traffic between plasma membrane, endosomes, Golgi/TGN membrane compartments within mammalian cells (Bastin et al., 2012). The ability of RGS4 to target different intracellular subdomains was highly dependent on the palmitoylation status of its amino-terminus. We showed that differential palmitoylation Cysteine2 and Cysteine12 had markedly different effects on its localization and function. Specifically, palmitoylation on Cys12 is critical for localization to the plasma membrane and inhibition of receptor-mediated Gprotein signaling, whereas palmitoylation on Cys 2 appears critical for allowing RGS4 to traffic into the intracellular endosome/Golgi pool. Together, these data suggested that RGS4 palmitoylation site mutants might be useful genetic tools for understanding the extent of plasma membrane versus intracellular G-protein activity that contributes to complex signaling, such as that by members of the MAPK family.

JNKs belong to the MAPK family. JNK isoforms have been implicated in the pathophysiology of a range of diseases, including Alzheimers disease (Akhter et al., 2015), arthritis (Schepetkin et al., 2015), obesity (Nakamura et al., 2015), diabetes (Dong et al., 2015), atherosclerosis (Kampschulte et al., 2014), abdominal aortic aneurysm (Zhang et al., 2015), cardiac disease (Sun et al., 2015), liver disease (Win et al., 2015), and tumorigenesis (Enomoto et al., 2015), making them an important target for therapeutic intervention. JNKs form part of a wider protein kinase cascade that mediates cellular responses to an array of stress-related stimuli including: heat shock (Ruan et al., 2015), hyperosmolarity (Gerke et al., 2014), ultraviolet irradiation (von Koschembahr et al., 2015), cytokine activity (Choi et al., 2015), and GPCRs (Yamauchi et al., 2000). Extracellular stress stimuli activate a cascade of kinases that involve mitogen-activated protein/ERK kinase kinase (MEKKs), mitogen-activated protein kinase kinase 4/7 (MKK4/7), and JNK1/2/3 assembled on intracellular scaffold proteins, such as JNK-interacting protein (JIP) (Whitmarsh, 2006). Once activated, JNKs can phosphorylate a large number of cellular proteins that are connected to stress signaling (Arthur and Ley, 2013). As their name suggests, JNKs are thought to be one of the most important activators of the transcription factor c-Jun via their ability to phosphorylate serines 63 and 73 within its transactivation domain (Li et al., 2004). JNKs may also activate other transcription factors, such as: JunD (Yazgan and Pfarr, 2002) and ATF2 (two members of the AP-1 transcription complex) (Gupta et al., 1995); hormone receptors (Caelles et al., 1997); FOXO4 (Essers et al., 2004); and PPAR $\gamma 1$ (Yin et al., 2006). Of importance to this work, JNKs have also been shown to phosphorylate a large number of protein substrates outside of the nucleus suggesting that they have important functions that are distinct from transcriptional regulation. Specifically, JNKs have been shown to regulate the following proteins in various compartments: cytoskeletal proteins - such as the microtubule-associated protein, tau (Yoshida et al., 2004); cytosolic proteins - including the E3 ligase, Itch (Gallagher et al., 2006), insulin-receptor substrate-1 (Hilder et al., 2003) and various 14-3-3 adaptors (Sunayama et al., 2005); mitochondrial proteins - such as Bcl2 (Yamamoto et al., 1999), Bad (Yu et al., 2004), and Bim (Lei and Davis, 2003); and plasma membrane-proximal components of focal adhesions - such as paxillin (Huang et al., 2003). It has been suggested that in order to phosphorylate such a wide variety of intracellular substrates, JNKs must be localized to the specific subcellular domains containing their substrates. Indeed, distinct pools of activated JNK have been identified in various intracellular compartments, including the nucleus (Kurinna et al., 2004), endosome/lysosome (Parameswaran et al., 2013), and 
mitochondrial fractions (Almeida et al., 2000); however, little is known about the neighborhood-specific molecules and pathways that are involved in regulating these unique signaling domains. We show here that the heterotrimeric G-protein, Goi3, and the intracellular proteins that regulate its activity, such as AGS3 and RGS4, represent a novel signaling compartment that controls the local activity levels of JNK. These data provide strong rationale for characterization of the relative contribution of different intracellular JNK pools to the overall JNK activity profile of a cell or tissue, particularly when evaluating the significance of changing phosho-JNK (p-JNK) levels on Western blots from whole cell lysates.

\section{Materials and Methods}

\section{Materials}

The RGS4-YFP, RGS4-YFP (Cys2A and Cys12A), and RGS4-YFP (EN-AA) mutants were described previously by our group (Bastin et al., 2012; Bastin and Heximer, 2013). The EN-AA mutation in the GAP domain of RGS4 (E87A, N88) renders the protein catalytically inactive by preventing its interaction with Goi (Srinivasa et al., 1998). HEK293 cells (tsA-201 derivative) were a kind gift from Zhong-Ping Feng (University of Toronto). All tissue culture media and transfection reagents were purchased from Invitrogen and Roche Scientific, respectively. Fluorescent-tagged versions of the TGN marker protein TGN38 were from J. LippincottSchwartz (National Institutes of Health, Bethesda, MD, USA). Goi3-CFP construct was created by insertion of CFP within an intracellular loop of Goi3 and was a kind gift from Catherine Berlot (Weis Center for Research, Geisinger Clinic, Danville, PA, USA). AGS3-GFP was a kind gift from Stephen Lanier (University of South Carolina). Antibodies against JNK and phospho-JNK were purchased from Cell Signaling with the respective catalog numbers: \#9252S, \#9251S. Horseradish peroxidase-coupled antirabbit secondary antibodies were from Cell Signaling (CAT \# 70745) respectively. Unless otherwise stated, all other reagents and chemicals were from Sigma.

\section{Cell Culture}

HEK293 or MEF cells were grown in Dulbecco's modified Eagle's medium (DMEM):Ham's F12 medium (1:1) (Gibco, respectively CAT \# 11995-065 and \# 11765-054), supplemented with 10\% (v/v) heat-inactivated fetal bovine serum (Gibco, CAT \# 12483020), $2 \mathrm{mM}$ glutamine (Life Technology, CAT \# 25030081), $10 \mu \mathrm{g} / \mathrm{ml}$ streptomycin, and $100 \mathrm{units} / \mathrm{ml}$ penicillin (Life Technology, CAT \# 15140122) at $37^{\circ} \mathrm{C}$ in a humidified atmosphere with $5 \% \mathrm{CO}_{2}$. To reduce the impact of growth factors and hormones on global cell signaling, cells were maintained in serum-free Earle's Balanced Sodium Solution (EBSS, Life Technology, CAT \#14155-063) supplemented with $200 \mathrm{mg} / \mathrm{L}$ of both $\mathrm{CaCl}_{2}$ and $\mathrm{MgCl}_{2}$.

\section{Molecular Biology}

For subcellular localization studies, RGS4-YFP and cysteine point mutations expression plasmids were generated in the pEYFP$\mathrm{C} 1$ as described previously (Bastin et al., 2012). Constitutively active Goi3-R178C-CFP was created by site-directed mutagenesis methods using the forward strand primers $5^{\prime}$-cca act cag cca gat gtt ctt cgg aca tgt- $3^{\prime}$ together with its reverse complement. The R178C mutation has been previously shown to impair the GTP hydrolysis activity by the $\alpha$ subunit, rendering the protein locked in its activated state. RGS proteins are however, capable of increasing the GTPase rate of these mutants. All plasmid constructs were purified using the Endofree Maxi kit (Qiagen, CAT \# 12362) and verified by sequencing of the complete protein-coding region.

\section{Confocal Microscopy}

HEK293 cells were plated at 50\% confluence in tissue culturetreated microscopy dishes (Ibidi, CAT \# 81156) and transfected overnight with $1 \mu \mathrm{g}$ of each construct to be tested using $2.5 \mu \mathrm{L}$ of Xtremegene HP transfection reagent according to the manufacturer's instructions (Roche, CAT \# 06366236001). After $24 \mathrm{~h}$, dishes were examined by confocal microscopy to determine their localization containing transfected cells. Spinning disk confocal microscopy was performed on live cells at $37^{\circ} \mathrm{C}$ in an environmental chamber maintained at $5 \% \mathrm{CO}_{2}$ using a WaveFX Spinning-Disk Confocal Microscope (Quorum Technologies, Guelph, Canada), comprised of an Olympus IX81 microscope stand, a Yokogawa CSU10 spinning-disk unit, and a Hamamatsu C9100-13 EM-CCD camera, controlled by Volocity software. Imaging was performed using a $60 \times / 1.42$ N.A. oil immersion objective, using 405, 488, and $561 \mathrm{~nm}$ solid-state lasers for the excitation of CFP, YFP, and mRFP respectively. Z-stack intervals were $0.3-0.35 \mu \mathrm{m}$. Emission wavelength parameters of each were matched to the appropriate bandpass emission filters, and where more than one fluorescent channel was examined in a single cell, the possibility of bleed through fluorescence was excluded prior to evaluation of the co-localization of different proteins. All confocal images were collected and analyzed with the Volocity software package and figures were subsequently generated using Microsoft Office.

\section{Western Blotting}

Proteins were transferred to (Trans-Blot, BioRad) nitrocellulose membrane. Membranes were blocked for $1 \mathrm{~h}$ with Tris-buffered saline $0.1 \%$ Tween-20 (TBST) with 5\% bovine serum albumin. Primary antibodies were diluted in TBST containing 5\% BSA as per the vendor's instructions and incubated with membranes overnight at $4^{\circ} \mathrm{C}$ before removing by washing. Horseradish peroxidase linked-secondary antibodies were diluted (1:3000) in TBST with 5\% BSA was added for $2 \mathrm{~h}$, before washing and signal detection using Super Signal West Pico Chemi-luminescent Substrate (Thermo Scientific). Western blots were analyzed by densitometry using Image J software analysis.

\section{Data Collection, Management, and Statistical Analysis}

At the outset of each series of microscopy experiments, the experimenter was blinded to the identity of the transfectants until data collection and analysis were completed. Where indicated, oneway and two-way ANOVA with Tukey's post hoc analysis were used to analyze the experimental results. ${ }^{\star} p<0.05$ was considered significant. Error bars depict standard error of the mean (SEM) for all graphs. 


\section{Results and Discussion}

Our previous work with the RGS4 protein showed that there exists at least two distinct membrane-bound pools of RGS4 within mammalian cells (Bastin et al., 2012). These pools consisted of the known pool at the plasma membrane and a newly appreciated pool that targeted intracellular membranes, such as endosomes, Golgi, and other punctate structures (Bastin and Heximer, 2013). The demonstration that differential palmitoylation of cysteine residues in the amino-terminus of RGS4 could alter its distribution between these two membrane pools guided efforts to demonstrate potential functional differences between RGS4 at these different locations in the cell. While it was relatively straightforward to show that prevention of RGS4 trafficking to the plasma membrane via mutation of Cys12, the palmitoylation site adjacent to its membrane-targeting amphipathic helix, could inhibit the ability of RGS4 to inhibit Gq-mediated signaling from the plasma membrane, it was more complicated to demonstrate a functional consequence of the Cys2 mutation that prevented RGS4 localization to the intracellular membrane pool. Our attention turned to regulation of intracellular Goi3 after we discovered overlapping expression of RGS4-YFP-containing punctae with those targeted by CFP-tagged Goi3 (Figure 1). Notably, the extent of co-localization on intracellular punctae was typically greater (larger number of punctae/cell) between RGS4 and the constitutively active Goi3 (R178C; RC) compared to Goi3 (WT). A significant plasma membrane signal
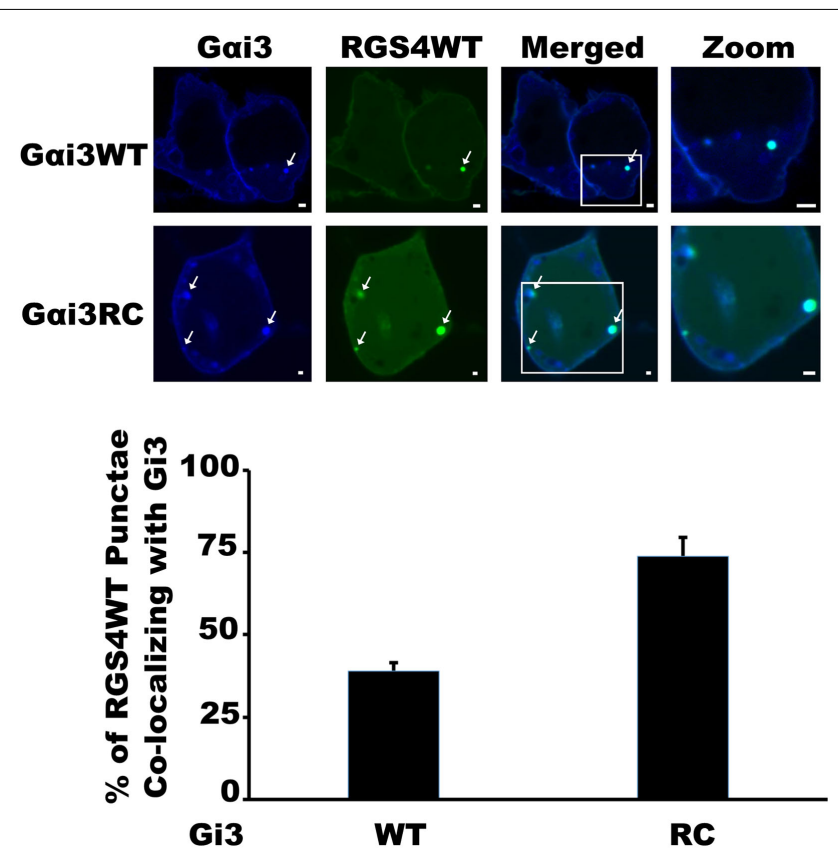

FIGURE 1 | RGS4 Shows preferential co-localization with G $\alpha$ i3RC HEK293 cells were co-transfected with RGS4-YFP (yellow), and either Goi3 (WT)- or Goi3 (RC)-CFP (blue) to assess the extent of co-localization using spinning disk confocal microscopy. The merged view is a composite two-channel view of cells expressing the two indicated constructs. Data are representative of at least 100 dual-transfected cells. Arrows indicate co-localization on intracellular endosomal structures between RGS4 and Goi3. Scale bars represent $1 \mu \mathrm{m}$.

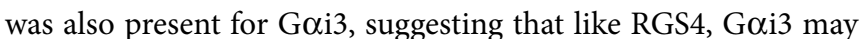
also traffic between the plasma membrane and the intracellular membrane compartments. These data suggested that intracellular RGS4 and Goi3 may target some of the same intracellular domains to co-ordinately regulate specific intracellular signaling

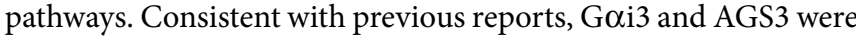
also found together on intracellular membrane structures in our expression system (Figure 2). Notably, in the presence of AGS3, we observed dramatically reduced co-localization of RGS4 and

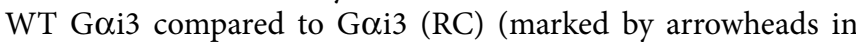
Figures 3A,B). Together, these data suggested that Goi3 may shuttle between AGS3-containing (GDP-bound Goi3) and RGS4containing (GTP-bound Goi3) compartments depending on its state of activation. These data simply reflect the preferences of the RGS box for GTP-bound Goi3 and AGS3 GPR/GoLoco motifs for inactive/GDP-bound Goi3; however, preliminary evidence to argue against this notion comes from experiments showing the catalytically dead RGS4 (EN-AA) mutant had similar colocalization with Goi3 (RC)-containing punctae as wild-type RGS4. Moreover, the co-expression of Goi3 with either RGS4 or AGS3 did not alter their localization in any discernable manner. These data suggest that these proteins traffic together on similar endosome-like structures where they may be co-localized, without necessarily interacting stably with one another. Such a system would allow RGS4 to fine tune the levels of Goi3 activity, while they are in the same compartment and then pass off inactive Goi3-GDP to another membrane compartment (presumably an AGS3-containing one), where Goi3 could be primed for reactivation.

We next examined JNK activity, an intracellular signaling pathway that we expected to be sensitive to changes in Goi3 activity. Indeed, the prediction, based on previous reports where JNK was activated downstream of various GPCRs, was that increased Goi3

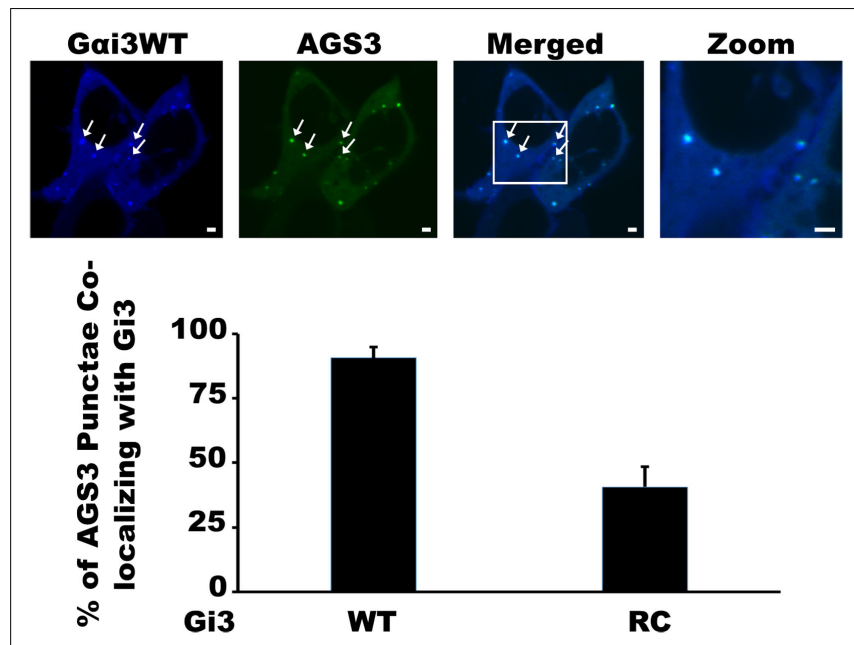

FIGURE 2 | Co-localization of Gai3 with AGS3 in mammalian cells. HEK 293 cells were co-transfected AGS3-GFP (green) and Gai3WT-CFP (blue) to assess the extent of co-localization using spinning disk confocal microscopy. The merged view is a composite two-channel view of cells expressing the two indicated constructs. Data are representative of at least 100 dual-transfected cells. Arrows indicate co-localization between AGS3 and Goi3 on intracellular endosomal structures. Scale bars represent $1 \mu \mathrm{m}$. 
A
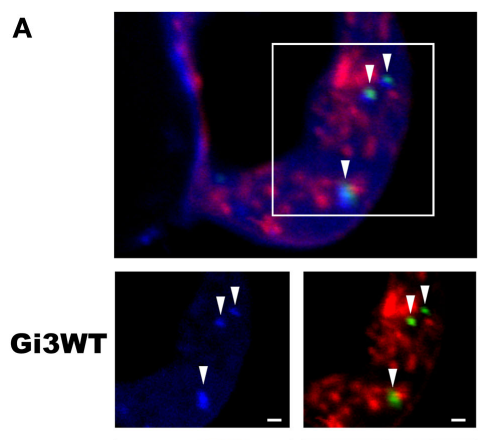

AGS3

RGS4
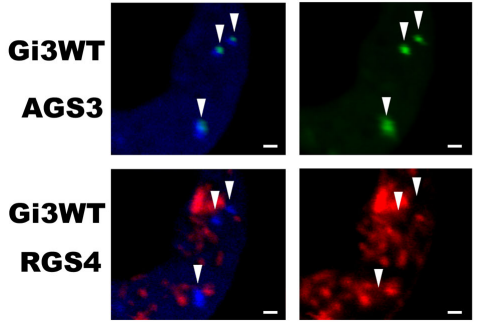

AGS3

FIGURE 3 | Co-localization of Gai3 with AGS3 and RGS4 is dependent on its activation state. HEK 293 cells were co-transfected with RGS4-RFP (red), AGS3-GFP (green), and either Goi3WT-CFP (A) or Goi3RC-CFP (B) (blue) to assess the extent of co-localization using spinning disk confocal microscopy. The merged view is a composite three-channel view of cells expressing all three
B
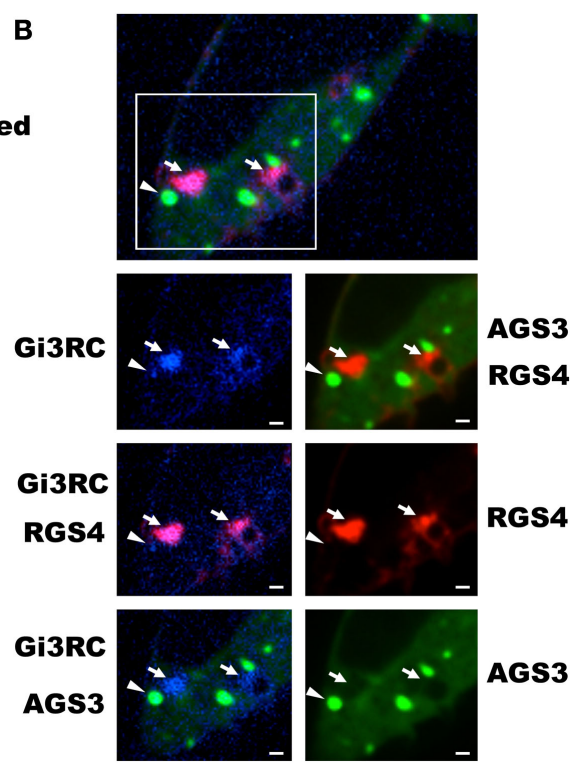

constructs. Shown below are the indicated single channel views or double-channel combinations. Data are representative of at least 100 triple-transfected cells. Arrows indicate co-localization between RGS4 and Goi3, whereas arrowheads indicate co-localization between AGS3 and Goi3. Scale bars represent $1 \mu \mathrm{m}$
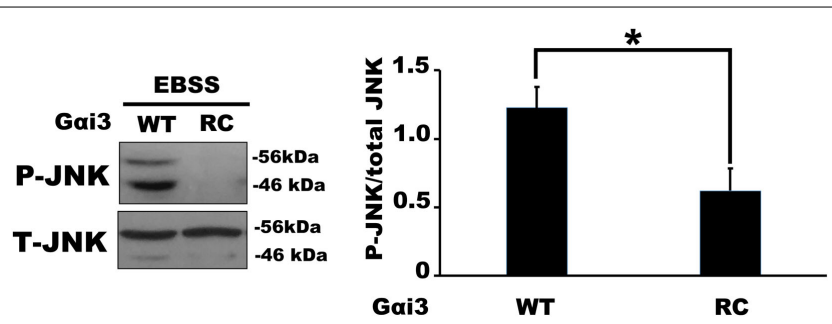

FIGURE 4 | Activated Gai3 reduces the levels of phospho(p)-JNK in HEK 293 cells. Relative changes in endogenous p-JNK/total JNK ratio were quantified in the presence of Goi3-CFP (WT) and (RC) by Western Blotting. The left panel show a representative Western blot of $p$-JNK/total JNK regulation by $\mathrm{G} \alpha \mathrm{i} 3(\mathrm{WT})$ and $(\mathrm{RC})$ expression. The right panel shows the quantification of $\mathrm{p}-\mathrm{JNK} /$ total $\mathrm{JNK}$ ratio in four independent experiments (one-way ANOVA with Tukey post hoc test ${ }^{*} P<0.01$ ).

activity would increase the level of intracellular JNK activation as determined by Western blotting for phosphorylated JNK ( $p$ -

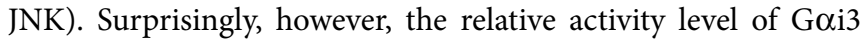
in our system inversely correlated with the observed p-JNK lev-

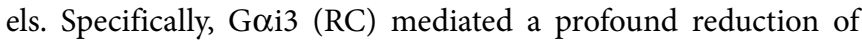
p-JNK in our system compared to Goi3 (WT) (Figure 4). As

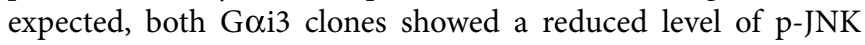
relative to empty vector controls (data not shown). Together, these data suggested the novel premise that Goi3 signaling inhibits intracellular JNK activation. Since, AGS3 functions as a GDI on intracellular membrane pools, we next examined whether AGS3mediated stabilization of GDP-bound (inactive) Goi3 may also regulate JNK activation. Consistent with our new model, AGS3 expression markedly increased the levels of p-JNK observed in our cells relative to YFP control (Figure 5). These data suggest that there exists a tonic level of endogenous Goi-mediated JNK inhibition in HEK293 cells that can be modulated by the expression of AGS3 or other similar GDI partners. In support of this, we also found that pertussis toxin increased p-JNK levels in a dosedependent fashion (data not shown). Finally, we examined the effect of another potent Goi3 inhibitor, RGS4, on the regulation of JNK activity in our system (Figure 5). Endogeneous Goi3 with wild-type RGS4 expression resulted in a modest decrease of pJNK compared to expression of its catalytically inactive EN-AA mutant. At first glance, these data seemed inconsistent with the

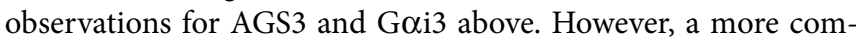
pelling story emerged when we examined the effects of the RGS4 palmitoylation site mutants Cys2A and Cys12A on intracellular JNK signaling. Notably, for the Cys2 mutant, when RGS4 was nearly exclusively localized to the plasma membrane (i.e., unable to target the intracellular membrane pool), there was a marked decrease in p-JNK levels. By contrast, for the Cys12A mutant when RGS4 was nearly exclusively localized to intracellular membranes, there was a marked increase in p-JNK levels to those even exceeding the levels observed for the catalytically inactive (ENAA) RGS4 construct. Taken together, these data suggest that total JNK signaling in a cell or tissue represents a combination of JNK pools that likely includes cytosolic, nuclear, plasma membrane, and intracellular fractions. Wild-type RGS4, by virtue of its ability to target and inhibit multiple intracellular signaling pools showed a much different effect on JNK signaling compared to either of the two individual palmitoylation site mutants. It should be noted, however, that we cannot rule out the possibility that mutation of Cys2 and its effects on RGS4 stability, via preventing N-end rule degradation of the protein may also have contributed to its ability to regulate intracellular JNK activity. A mechanistic model 
A

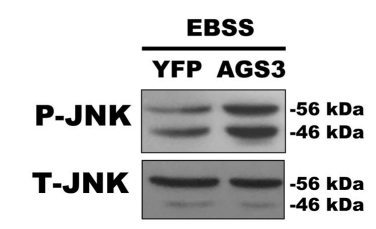

B

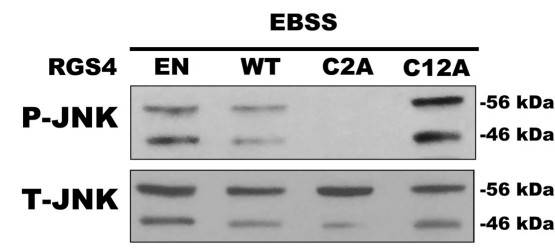

FIGURE 5 | Expression of AGS3 and intracellular RGS4 increased phospho(p)-JNK in HEK 293 cells. (A) Relative changes in endogenous $p-J N K / t o t a l$ JNK ratio were quantified in the presence of AGS3 or empty vector as indicated. The left panel shows a representative Western blot of $p-J N K / t o t a l$ JNK regulation by AGS3 expression. The right panel shows the quantification of p-JNK/total JNK ratio in four independent experiments. (B) The effect of RGS activity of RGS4 on p-JNK/total JNK was observed by Western blot with the
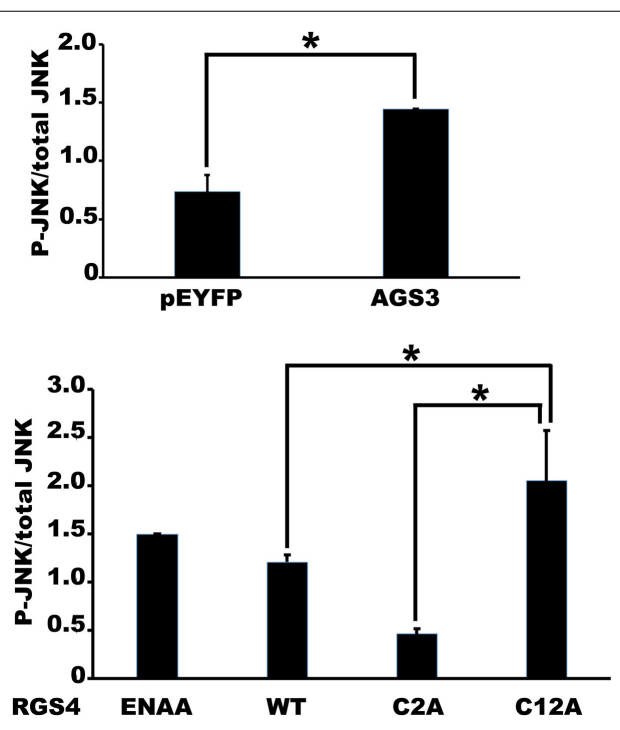

catalytic dead mutant RGS4 (ENAA). The effect of palmitoylation site of RGS4 on the variation of $\mathrm{p}-\mathrm{JNK} /$ total JNK was observed by Western blot with the expression of Cys2 and Cys12 mutants. The left panel shows a representative Western blot of $\mathrm{p}$-JNK/total JNK regulation by the indicated constructs. The right panel shows the quantification of $\mathrm{p}-\mathrm{JNK} /$ total $\mathrm{JNK}$ ratio in four independent experiments [one-way ANOVA with post hoc Tukey ${ }^{*} P<0.005$ for $(\mathbf{A})$, and ${ }^{\star} P<0.05$ for $\left.(\mathbf{B})\right]$. showing the spatial distribution of JNK, G $\alpha \mathrm{i} 3$, and its regulators is presented in Figure 6.

It is important to note that the mechanism by which Goi3 inhibits intracellular JNK remains to be identified. It will be critical to determine whether this unique effect is mediated by

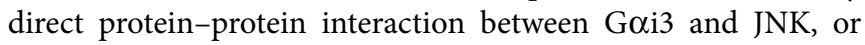
alternatively whether other effector pathways downstream of activated Goi3, such as adenylyl cyclases or PI3Ks, may be involved. Although the effects of Goi3 (RC) are consistent with an effect mediated by the $\alpha$ subunit, further studies will be required to determine whether $\beta \gamma$ or other $\beta \gamma$-like partners may also participate in the complexes that regulate intracellular JNK activity. Moreover, while it appears likely that the effects of AGS3 and

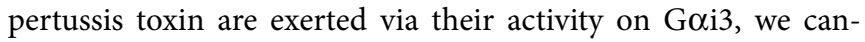
not rule out the possibility that other endogenous G $\alpha$ i subunits may also contribute to intracellular JNK regulation. These new findings may also be useful in the design of strategies to identify and selectively target specific intracellular JNK pools and their associated physiologic activities. At this stage, it remains to be determined whether the JNK pool regulated by Goi3 in our system is associated with one of the known regulatory functions of JNK, such as apoptosis, cell migration, transcription, autophagy, and ER-Golgi trafficking, or whether it has a yet undiscovered function. It seems likely, however, based on the

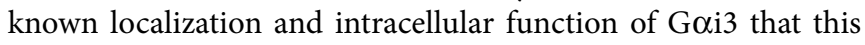
pool of JNK will be somehow linked to intracellular membrane trafficking at the level of the ER-Golgi and their associated vesicular compartments.

It may be important to determine whether this signaling domain contains one or more of the different known JNK isoforms. Mammalian JNKs are encoded by three distinct genes
(Jnk1, Jnk2, and Jnk3). Alternative splicing generates up to 10 different protein products varying in size from 46 to $55 \mathrm{kDa}$, all of which have been sequenced and analyzed for possible specificity determinants. Despite their the high level of homology $(>80 \%)$, differences between amino- and carboxy-terminal sequence or exon usage suggest the existence of functional specificity (Gupta et al., 1996; Guo and Whitmarsh, 2008). While isoform-specific knockouts and the development of pan-specific JNK inhibitors have thus far been very useful in the study of JNK function (Gehringer et al., 2015), the simple fact remains that for most JNK substrates, there is still very little information regarding isoformspecific affinity. As suggested by our data above, targeting discreet intracellular protein complexes, such as endosomal Goi3, or its regulators and effectors, may offer unique molecular strategies for modulating JNK activity. One such strategy might be to alter the plasma membrane versus endosomal distribution profile of the Goi3 regulator RGS4. This might be accomplished by promoting site-selective palmitoylation of its amino-terminus. Currently, studies are underway to evaluate the specificity of the various palmitoyl-CoA transferases (DHHC family proteins) for Cys2 and Cys12 with this in mind. It is also interesting that the aminoterminal domain of G $\alpha$ i3 itself requires palmitoylation for its optimal membrane targeting and function. Thus, regulation of Goi3 by specific DHHC isoforms might provide another useful access point for regulating the intracellular JNK pool. Lastly, modulation of AGS3 activity may provide another unique opportunity to modulate intracellular JNK via Goi3. Using structural techniques and data, other groups have identified the residues within AGS3 that allow its binding (Peterson et al., 2002; Willard et al., 2008) and GDI activity (Kimple et al., 2002, 2004) for Goi. Intriguingly, these data may ultimately inform the design of peptide mimetics 


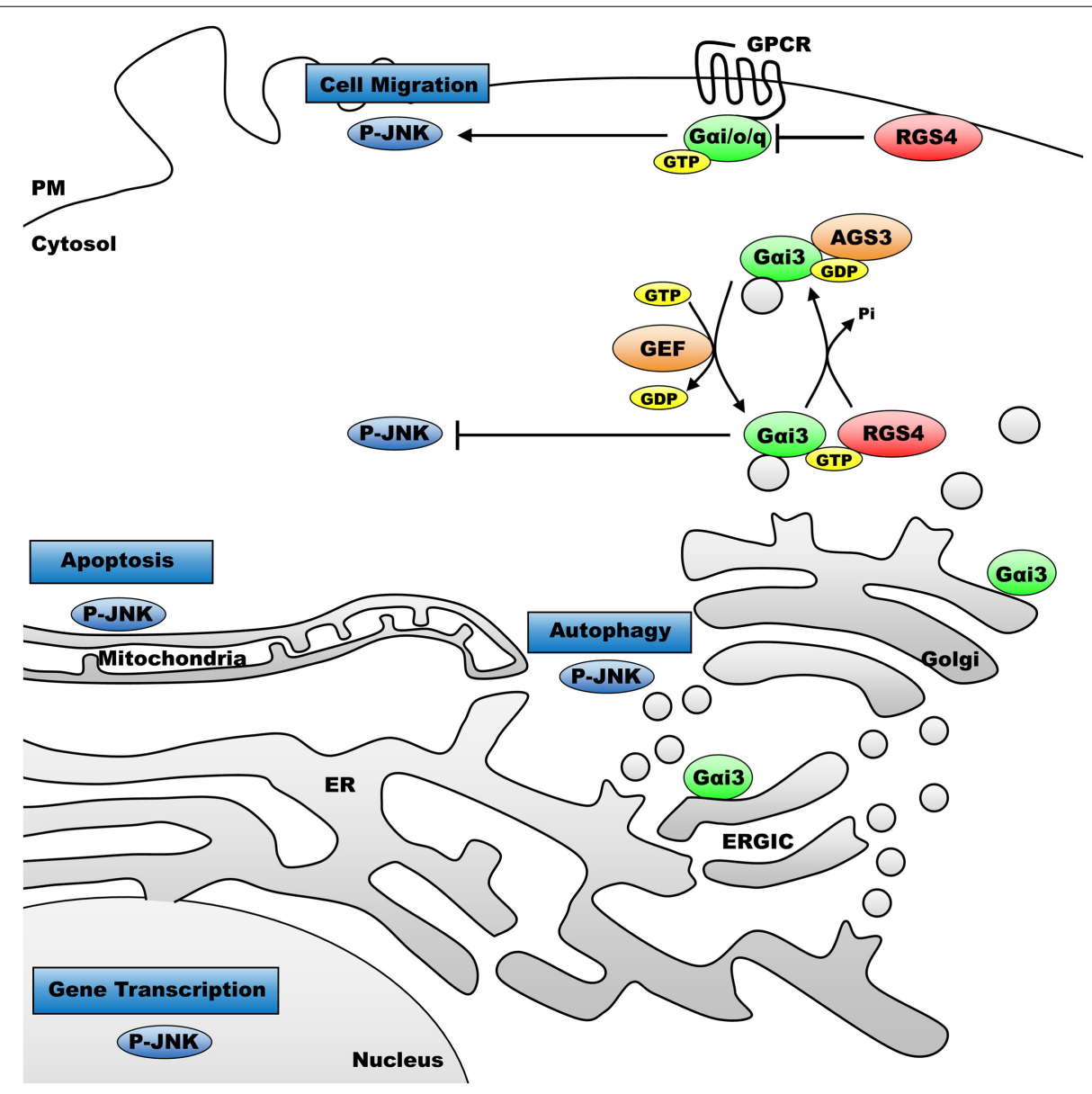

FIGURE 6 | Model for intracellular functions of $p-J N K$ and the effect of heterotrimeric G-proteins on p-JNK accordingly to the cellular compartment. P-JNK has been correlated to distinct functions accordingly to its intracellular compartments. Indeed, p-JNK regulates gene transcription upon nuclear localization, apoptosis when it targets mitochondria. P-JNK also regulates signals located at the ER-Golgi vicinity to enhance autophagy and at the plasma membrane to regulate cell migration. Heterotrimeric G-protein coupled receptor activation at the plasma membrane is known to increase levels of $\mathrm{p}$-JNK in mammalian cells. Here, we showed that activated Goi3 protein significantly inhibits p-JNK and inhibitors of Goi/o proteins, AGS3 and RGS4, promote p-JNK when located on intracellular compartments. Conversely, RGS4 located selectively at the plasma membrane potently inhibited p-JNK. that selectively could interfere with AGS3-Goi3 interactions to

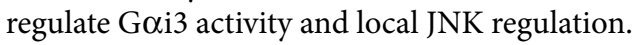

In summary, we have uncovered a new role for Goi3-mediated function in mammalian cells - specifically as an inhibitor of JNK activation and signaling. The model for the proposed mechanism is shown in Figure 6. Activation of Goi3 (in this case via receptor-independent GEF activity) on intracellular membrane pools leads to inhibition of local JNK activation. Then, illustrated, the regulation G $\alpha \mathrm{i} 3$ activity (cycling between different nucleotide states) may be coordinated by other intracellular players, including AGS3, RGS proteins, and GIV/girdin (GEF). Changes in intracellular JNK activity may then alter physiologic activities at one or more of the other intracellular locations where unique JNK pools are thought to be important, such as mitochondria (apoptosis), ER-Golgi interface (autophagy), or the nucleus (transcriptional programs). This work supports further characterization of the novel protein complexes regulated by heterotrimeric G-proteins that are found localized to intracellular membrane pools deep within the cell, distal to the influences of most surface GPCRs. The characterization of receptor-independent G-protein activation and signaling, and its relationship to cellular health and homeostasis is an important emerging area of investigation that is likely to reveal a number of new cellular functions for $\mathrm{G} \alpha$ subunits, as well as their intracellular binding partners and effectors.

\section{Acknowledgments}

We are grateful to Fakhriyeh Abachi Nejad Asl for technical assistance on this project. Guillaume Bastin was funded by a Postdoctoral Research Fellowship from the Richard Lewar Heart and Stroke Association Centre of Excellence in Cardiovascular Research. Project support for this work came from Operating Grants from the Canadian Institutes of Health Research (MOP106670) and the Grants-in-Aid Program of the Heart and Stroke Foundation of Canada (T6799). 


\section{References}

Akhter, R., Sanphui, P., Das, H., Saha, P., and Biswas, S. C. (2015). The regulation of p53 up-regulated modulator of apoptosis by JNK/c-Jun pathway in beta Amyloid-induced neuron death. J. Neurochem. doi:10.1111/jnc.13128

Almeida, E. A., Ilic, D., Han, Q., Hauck, C. R., Jin, F., Kawakatsu, H., et al. (2000). Matrix survival signalling: from fibronectin via focal adhesion kinase to $\mathrm{c}-J u n$ $\mathrm{NH}$ (2)-terminal kinase. J. Cell Biol. 149, 741-754. doi:10.1083/jcb.149.3.741

Arthur, J. S., and Ley, S. C. (2013). Mitogen-activated protein kinases in innate immunity. Nat. Rev. Immunol. 13, 679-692. doi:10.1038/nri3495

Bastin, G., and Heximer, S. (2013). Rab family protein regulate the endosomal trafficking and function of RGS4. J. Biol. Chem. 288, 21836-21849. doi:10.1074/ jbc.M113.466888

Bastin, G., Singh, K., Dissanayake, K., Mighiu, A. S., Nurmohamed, A., and Heximer, S. P. (2012). Amino-terminal cysteine residues differentially influence RGS4 protein plasma membrane targeting, intracellular trafficking, and function. J. Biol. Chem. 287, 28966-28974. doi:10.1074/jbc.M112.345629

Berman, D. M., Wilkie, T. M., and Gilman, A. G. (1996). GAIP and RGS4 are GTPase-activating proteins for the Gi subfamily of $G$ protein alpha subunits. Cell 86, 445-452. doi:10.1016/S0092-8674(00)80117-8

Caelles, C., Gonzalez-Sancho, J. M., and Munoz, A. (1997). Nuclear hormone receptor antagonism with AP-1 by inhibition of the JNK pathway. Genes Dev. 11, 3351-3364. doi:10.1101/gad.11.24.3351

Choi, H., Dikalova, A., Stark, R. J., and Lamb, F. S. (2015). c-Jun N-terminal kinase attenuates TNFalpha signalling by reducing Nox1-dependent endosomal ROS production in vascular smooth muscle cells. Free Radic. Biol. Med. 86, 219-227. doi:10.1016/j.freeradbiomed.2015.05.015

De Vries, L., Elenko, E., McCaffery, J. M., Fischer, T., Hubler, L., McQuistan, T., et al. (1998). RGS-GAIP, a GTPase-activating protein for Galphai heterotrimeric G proteins, is located on clathrin-coated vesicles. Mol. Biol. Cell 9, 1123-1134. doi:10.1091/mbc.9.3.599

Dong, Y., Gao, G., Fan, H., Li, S., Li, X., and Liu, W. (2015). Activation of the liver X receptor by agonist TO901317 improves hepatic insulin resistance via suppressing reactive oxygen species and JNK pathway. PLoS ONE 10:e0124778. doi:10.1371/journal.pone. 0124778

Enomoto, M., Kizawa, D., Ohsawa, S., and Igaki, T. (2015). JNK signalling is converted from anti- to pro-tumor pathway by Ras-mediated switch of Warts activity. Dev. Biol. 403, 162-171. doi:10.1016/j.ydbio.2015.05.001

Essers, M. A., Weijzen, S., de Vries-Smits, A. M., Saarloos, I., de Ruiter, N. D., Bos, J. L., et al. (2004). FOXO transcription factor activation by oxidative stress mediated by the small GTPase Ral and JNK. EMBO J. 23, 4802-4812. doi:10. 1038/s.emboj.7600476

Gallagher, E., Gao, M., Liu, Y. C., and Karin, M. (2006). Activation of the E3 ubiquitin ligase itch through a phosphorylation-induced conformational change. Proc. Natl. Acad. Sci. U.S.A. 103, 1717-1722. doi:10.1073/pnas.0510664103

Garcia-Marcos, M., Ear, J., Farquhar, M. G., and Ghosh, P. (2011). A GDI (AGS3) and a GEF (GIV) regulate autophagy by balancing $\mathrm{G}$ protein activity and growth factor signals. Mol. Biol. Cell 22, 673-686. doi:10.1091/mbc.E10-08-0738

Gehringer, M., Muth, F., Koch, P., and Laufer, S. A. (2015). c-Jun N-terminal kinase inhibitors: a patent review (2010-2014). Expert Opin. Ther. Pat. 25, 849-872. doi:10.1517/13543776.2015.1039984

Gerke, P., Keshet, A., Mertenskotter, A., and Paul, R. J. (2014). The JNK-like MAPK KGB-1 of Caenorhabditis elegans promotes reproduction, lifespan, and gene expressions for protein biosynthesis and germline homeostasis but interferes with hyperosmotic stress tolerance. Cell. Physiol. Biochem. 34, 1951-1973. doi: $10.1159 / 000366392$

Guo, C., and Whitmarsh, A. J. (2008). The beta-arrestin-2 scaffold protein promotes c-Jun N-terminal kinase- 3 activation by binding to its nonconserved $\mathrm{N}$ terminus. J. Biol. Chem. 283, 15903-15911. doi:10.1074/jbc.M710006200

Gupta, S., Barrett, T., Whitmarsh, A. J., Cavanagh, J., Sluss, H. K., Derijard, B., et al. (1996). Selective interaction of JNK protein kinase isoforms with transcription factors. $E M B O$ J. 15, 2760-2770.

Gupta, S., Campbell, D., Derijard, B., and Davis, R. J. (1995). Transcription factor ATF2 regulation by the JNK signal transduction pathway. Science 267, 389-393. doi:10.1126/science.7824938

Hilder, T. L., Tou, J. C., Grindeland, R. E., Wade, C. E., and Graves, L. M. (2003). Phosphorylation of insulin receptor substrate-1 serine 307 correlates with JNK activity in atrophic skeletal muscle. FEBS Lett. 553, 63-67. doi:10.1016/S00145793(03)00972-4
Hollinger, S., and Hepler, J. R. (2002). Cellular regulation of RGS proteins: modulators and integrators of $\mathrm{G}$ protein signalling. Pharmacol. Rev. 54, 527-559. doi:10.1124/pr.54.3.527

Hopkins, A. L., and Groom, C. R. (2002). The druggable genome. Nat. Rev. Drug Discov. 1, 727-730. doi:10.1038/nrd892

Huang, C., Rajfur, Z., Borchers, C., Schaller, M. D., and Jacobson, K. (2003). JNK phosphorylates paxillin and regulates cell migration. Nature 424, 219-223. doi: 10.1038/nature 01745

Kampschulte, M., Stockl, C., Langheinrich, A. C., Althohn, U., Bohle, R. M., Krombach, G. A., et al. (2014). Western diet in ApoE-LDLR double-deficient mouse model of atherosclerosis leads to hepatic steatosis, fibrosis, and tumorigenesis. Lab. Invest. 94, 1273-1282. doi:10.1038/labinvest.2014.112

Kimple, R. J., Kimple, M. E., Betts, L., Sondek, J., and Siderovski, D. P. (2002). Structural determinants for GoLoco-induced inhibition of nucleotide release by Galpha subunits. Nature 416, 878-881. doi:10.1038/416878a

Kimple, R. J., Willard, F. S., Hains, M. D., Jones, M. B., Nweke, G. K., and Siderovski, D. P. (2004). Guanine nucleotide dissociation inhibitor activity of the triple GoLoco motif protein G18: alanine-to-aspartate mutation restores function to an inactive second GoLoco motif. Biochem. J. 378(Pt 3), 801-808. doi:10.1042/ bj20031686

Kurinna, S. M., Tsao, C. C., Nica, A. F., Jiffar, T., and Ruvolo, P. P. (2004). Ceramide promotes apoptosis in lung cancer-derived A 549 cells by a mechanism involving c-Jun NH2-terminal kinase. Cancer Res. 64, 7852-7856. doi:10.1158/0008-5472. CAN-04-1552

Lei, K., and Davis, R. J. (2003). JNK phosphorylation of Bim-related members of the Bcl2 family induces Bax-dependent apoptosis. Proc. Natl. Acad. Sci. U.S.A. 100, 2432-2437. doi:10.1073/pnas.2332656100

Li, L., Feng, Z., and Porter, A. G. (2004). JNK-dependent phosphorylation of c-Jun on serine 63 mediates nitric oxide-induced apoptosis of neuroblastoma cells. J. Biol. Chem. 279, 4058-4065. doi:10.1074/jbc.M400525200

Lo, I. C., Gupta, V., Midde, K. K., Taupin, V., Lopez-Sanchez, I., Kufareva, I., et al. (2015). Activation of Galphai at the Golgi by GIV/girdin imposes finiteness in Arf1 signalling. Dev. Cell 33, 189-203. doi:10.1016/j.devcel.2015. 02.009

Nakamura, T., Kunz, R. C., Zhang, C., Kimura, T., Yuan, C. L., Baccaro, B., et al. (2015). A critical role for PKR complexes with TRBP in immunometabolic regulation and eIF2alpha phosphorylation in obesity. Cell Rep. 11, 295-307. doi:10.1016/j.celrep.2015.03.021

Oner, S. S., Vural, A., and Lanier, S. M. (2013). Translocation of activator of Gprotein signalling 3 to the Golgi apparatus in response to receptor activation and its effect on the trans-Golgi network. J. Biol. Chem. 288, 24091-24103. doi:10.1074/jbc.M112.444505

Parameswaran, N., Enyindah-Asonye, G., Bagheri, N., Shah, N. B., and Gupta, N. (2013). Spatial coupling of JNK activation to the B cell antigen receptor by tyrosine-phosphorylated ezrin. J. Immunol. 190, 2017-2026. doi:10.4049/ jimmunol.1201292

Peterson, Y. K., Hazard, S. III, Graber, S. G., and Lanier, S. M. (2002). Identification of structural features in the G-protein regulatory motif required for regulation of heterotrimeric G-proteins. J. Biol. Chem. 277, 6767-6770. doi:10.1074/jbc. C100699200

Ruan, J., Qi, Z., Shen, L., Jiang, Y., Xu, Y., Lan, L., et al. (2015). Crosstalk between JNK and NF-kappaB signalling pathways via HSP27 phosphorylation in HepG2 cells. Biochem. Biophys. Res. Commun. 456, 122-128. doi:10.1016/j.bbrc.2014. 11.045

Schepetkin, I. A., Kirpotina, L. N., Hammaker, D., Kochetkova, I., Khlebnikov, A. I., Lyakhov, S. A., et al. (2015). Anti-inflammatory effects and joint protection in collagen-induced arthritis after treatment with IQ-1S, a selective c-Jun $\mathrm{N}$ terminal kinase inhibitor. J. Pharmacol. Exp. Ther. 353, 505-516. doi:10.1124/ jpet.114.220251

Siderovski, D. P., and Willard, F. S. (2005). The GAPs, GEFs, and GDIs of heterotrimeric G-protein alpha subunits. Int. J. Biol. Sci. 1, 51-66. doi:10.7150/ ijbs.1.51

Srinivasa, S. P., Watson, N., Overton, M. C., and Blumer, K. J. (1998). Mechanism of RGS4, a GTPase-activating protein for G protein alpha subunits. J. Biol. Chem. 273, 1529-1533. doi:10.1074/jbc.273.3.1529

Sun, F., Duan, W., Zhang, Y., Zhang, L., Qile, M., Liu, Z., et al. (2015). Simvastatin alleviates cardiac fibrosis induced by infarction via up-regulation of transforming growth factor, beta receptor III expression. Br. J. Pharmacol. 172, 3779-3792. doi:10.1111/bph.13166 
Sunayama, J., Tsuruta, F., Masuyama, N., and Gotoh, Y. (2005). JNK antagonizes Akt-mediated survival signals by phosphorylating 14-3-3. J. Cell Biol. 170, 295-304. doi:10.1083/jcb.200409117

von Koschembahr, A. M., Swope, V. B., Starner, R. J., and Abdel-Malek, Z. A. (2015). Endothelin-1 protects human melanocytes from UV-induced DNA damage by activating JNK and p38 signalling pathways. Exp. Dermatol. 24, 269-274. doi:10.1111/exd.12638

Vural, A., Oner, S., An, N., Simon, V., Ma, D., Blumer, J. B., et al. (2010). Distribution of activator of G-protein signalling 3 within the aggresomal pathway: role of specific residues in the tetratricopeptide repeat domain and differential regulation by the AGS3 binding partners Gi(alpha) and mammalian inscuteable. Mol. Cell. Biol. 30, 1528-1540. doi:10.1128/MCB.01018-09

Whitmarsh, A. J. (2006). The JIP family of MAPK scaffold proteins. Biochem. Soc. Trans. 34(Pt 5), 828-832. doi:10.1042/BST0340828

Willard, F. S., Zheng, Z., Guo, J., Digby, G. J., Kimple, A. J., Conley, J. M., et al. (2008). A point mutation to Galphai selectively blocks GoLoco motif binding: direct evidence for Galpha.GoLoco complexes in mitotic spindle dynamics. J. Biol. Chem. 283, 36698-36710. doi:10.1074/jbc.M804936200

Win, S., Than, T. A., Le, B. H., Garcia-Ruiz, C., Fernandez-Checa, J. C., and Kaplowitz, N. (2015). Sab (Sh3bp5) dependence of JNK mediated inhibition of mitochondrial respiration in palmitic acid induced hepatocyte lipotoxicity. J. Hepatol. 62, 1367-1374. doi:10.1016/j.jhep.2015.01.032

Yamamoto, K., Ichijo, H., and Korsmeyer, S. J. (1999). BCL-2 is phosphorylated and inactivated by an ASK1/Jun N-terminal protein kinase pathway normally activated at G(2)/M. Mol. Cell. Biol. 19, 8469-8478.

Yamauchi, J., Kawano, T., Nagao, M., Kaziro, Y., and Itoh, H. (2000). Gi-dependent activation of c-Jun N-terminal kinase in human embryonal kidney 293 cells. J. Biol. Chem. 275, 7633-7640. doi:10.1074/jbc.275.11.7633
Yazgan, O., and Pfarr, C. M. (2002). Regulation of two JunD isoforms by Jun Nterminal kinases. J. Biol. Chem. 277, 29710-29718. doi:10.1074/jbc.M204552200

Yin, R., Dong, Y. G., and Li, H. L. (2006). PPARgamma phosphorylation mediated by JNK MAPK: a potential role in macrophage-derived foam cell formation. Acta Pharmacol. Sin. 27, 1146-1152. doi:10.1111/j.1745-7254.2006.00359.x

Yoshida, H., Hastie, C. J., McLauchlan, H., Cohen, P., and Goedert, M. (2004). Phosphorylation of microtubule-associated protein tau by isoforms of c-Jun N-terminal kinase (JNK). J. Neurochem. 90, 352-358. doi:10.1111/j.1471-4159. 2004.02479.x

Yu, C., Minemoto, Y., Zhang, J., Liu, J., Tang, F., Bui, T. N., et al. (2004). JNK suppresses apoptosis via phosphorylation of the proapoptotic $\mathrm{Bcl}-2$ family protein BAD. Mol. Cell 13, 329-340. doi:10.1016/S1097-2765(04)00083-8

Zhang, X. J., He, C., Tian, K., Li, P., Su, H., and Wan, J. B. (2015). Ginsenoside $\mathrm{Rb} 1$ attenuates angiotensin II-induced abdominal aortic aneurysm through inactivation of the JNK and p38 signalling pathways. Vascul. Pharmacol. doi:10. 1016/j.vph.2015.04.003

Conflict of Interest Statement: The authors declare that the research was conducted in the absence of any commercial or financial relationships that could be construed as a potential conflict of interest.

Copyright $\odot 2015$ Bastin, Yang and Heximer. This is an open-access article distributed under the terms of the Creative Commons Attribution License (CC BY). The use, distribution or reproduction in other forums is permitted, provided the original author(s) or licensor are credited and that the original publication in this journal is cited, in accordance with accepted academic practice. No use, distribution or reproduction is permitted which does not comply with these terms. 